\title{
Distributed Adaptive Consensus and Output Tracking of Unknown Linear Systems on Directed Graphs *
}

\author{
Zhongkui Li ${ }^{\mathrm{a}}$, Zhengtao Ding ${ }^{\mathrm{b}}$ \\ ${ }^{a}$ State Key Laboratory for Turbulence and Complex Systems, Department of Mechanics and Engineering Science, College of \\ Engineering, Peking University, Beijing 100871, China \\ ${ }^{\mathrm{b}}$ Control Systems Centre, School of Electrical and Electronic Engineering, University of Manchester, Sackville Street Building, \\ Manchester M13 9PL, UK
}

\begin{abstract}
This paper considers both the distributed consensus control and output tracking problems of a group of unknown linear subsystems using the relative output information of neighboring subsystems. Each system is a minimum-phase SISO system with relative degree one and unknown parameters, and the interaction graph among the subsystems is directed. For the case where the directed graph is strongly connected, a distributed adaptive protocol is designed to achieve consensus. For the case where there exists an arbitrary constant reference signal, a distributed adaptive controller together with an internal model are presented to achieve output tracking in the sense that the subsystem outputs asymptotically follow a reference constant. The proposed adaptive protocols are independent of the parameters of the subsystems, only use the relative outputs of neighboring subsystems, and hence are fully distributed.
\end{abstract}

Key words: Consensus control, output tracking, adaptive control, internal model, output feedback.

\section{Introduction}

In the last decade, consensus control of a group of autonomous subsystems (usually called multi-agent systems) has been an emerging research topic in the systems and control community. Consensus means that a team of subsystems reaches an agreement on a common value by interacting with their local neighbors. Due to its potential applications in several areas such as spacecraft formation flying, sensor networks, and cooperative surveillance [18], the consensus control problem has been addressed by many researchers from various perspectives; see $[17,18,4,14,1,3,24]$ and the references therein. Existing consensus algorithms can be roughly categorized into two classes, namely, consensus without a leader (i.e., leaderless consensus) and consensus with a leader which is also called leader-follower consensus or

\footnotetext{
* This work was supported by the National Natural Science Foundation of China under grants 61473005, 11332001, a Foundation for the Author of National Excellent Doctoral Dissertation of PR China, 111 Project (B08015), and the State Key Laboratory of Complex System Intelligent Control and Decision.
}

Email addresses: zhongkli@pku.edu.cn (Zhongkui Li), zhengtao.ding@manchester.ac.uk (Zhengtao Ding). distributed tracking.

For a consensus control problem, a key task is to design appropriate distributed consensus protocols to achieve consensus, using only the local state or output information of the subsystems and their neighbors. Note that designing distributed protocols is generally a challenging task, especially for the case with complex subsystem dynamics, due to the interplay of the subsystem dynamics, the interactions among subsystems, and those protocols. In most existing works, e.g., $[16,8,7,21,19,23,12]$, the design of the consensus protocols requires the knowledge of the nonzero eigenvalue information of the Laplacian matrix associated with the interaction graph. However, the nonzero eigenvalues of the Laplacian matrix are global information in the sense that each subsystem has to know the entire communication graph $\mathcal{G}$ to compute them. Therefore, the consensus protocols given in the aforementioned papers cannot be designed by each subsystem in a fully distributed way, i.e., using only the local information of its own and neighbors. Fully distributed adaptive consensus protocols are proposed in [10,9], which implement adaptive coupling weights to deal with the effect of the nonzero eigenvalues of the Laplacian matrix on consensus. Similar adaptive schemes are presented to achieve second-order consensus with nonlinear dy- 
namics in $[20,22]$. Note that the protocols in $[10,9,20,22]$ are applicable to only undirected communication graphs or leader-follower graphs where the subgraphs among the followers are undirected. Designing distributed adaptive consensus protocols for the case with general directed graphs is much more challenging, due to the asymmetry of the corresponding Laplacian matrices. By carefully choosing an integral-type Lyapunov function, a distributed adaptive consensus protocol is constructed in [11] to achieve leader-follower consensus for any communication graph containing a directed spanning tree with the leader as the root node. It is worth mentioning that the adaptive protocol in [11] relies on the relative states of neighboring subsystems. How to design adaptive consensus protocols using the relative output information for linear subsystems with directed graphs is much more challenging and to the knowledge of the authors is still open. Moreover, in $[10,9,20,22,11]$, the subsystem dynamics are assumed to be precisely known, by using which the adaptive consensus protocols are determined. However, in many circumstances, the subsystems may contain unknown parameters or uncertainties.

In this paper, we consider the distributed consensus control problem of a group of unknown linear subsystem$\mathrm{s}$ using the relative output information of neighboring subsystems. Each subsystem is a minimum-phase SISO system with relative degree one and unknown parameters, and the interaction graph among the subsystems is directed. Both leaderless consensus and distributed output tracking are considered. For the case where the interaction graph among the subsystems is strongly connected, a distributed adaptive protocol is designed to achieve leaderless consensus. For the case where there exists an unknown constant reference signal, a distributed adaptive controller together with an internal model are presented to achieve output tracking in the sense that the subsystem outputs asymptotically follow the given reference constant, if the interaction graph among the subsystems is strongly connected and at least one subsystem has access to the reference signal. Compared to the previous related works $[10,9,20,22,11]$, the contribution of this paper is at least two-fold. First, contrary to subsystems in $[10,9,20,22,11]$ whose dynamics are precisely known, the parameters of the subsystems are unknown in this paper. Second, in contrast to the adaptive protocol in [11] which relies on the relative states of neighboring subsystems, the adaptive protocols proposed in this paper depend on only the relative output information, which clearly is more challenging.

The rest of this paper is organized as follows. The leaderless consensus problem of a group of unknown linear subsystems with strongly connected directed graphs is addressed in Section 2. The distributed output tracking in the presence of a constant reference signal is considered in Section 3. A simulation example is presented for illustration in Section 4. Conclusions are drawn in Section 5 .

\section{Consensus of Unknown Linear Systems with Strongly Connected Directed Graphs}

\subsection{Problem Statement}

In this section, we consider a set of $N$ unknown linear subsystems (i.e., agents), of which the dynamics of the $i$-th subsystem are described by

$$
\begin{aligned}
& \dot{y}_{i}=h^{T} z_{i}+a y_{i}+b u_{i}, \\
& \dot{z}_{i}=B z_{i}+d y_{i}, \quad i=1, \cdots N
\end{aligned}
$$

where $z_{i} \in \mathbb{R}^{n-1}$ is the internal state vector, with $n$ a known positive constant integer denoting the order of the subsystems, $y_{i}, u_{i} \in \mathbb{R}$ are the output and input of the $i$-th subsystem, respectively, $B \in \mathbb{R}^{(n-1) \times(n-1)}$ is an unknown Hurwitz matrix, $d, h \in \mathbb{R}^{n-1}$ are unknown constant vectors, and $a, b \in \mathbb{R}$ are unknown constants, of which $b$ is also known as the high-frequency gain of the subsystems.

We make the following assumption about the dynamics of the subsystems.

Assumption 1 The sign of the high-frequency gain $b$ is known. Without lost of generality, it is further assumed that $b>0$.

Remark 1 It can be shown that any controllable and observable minimum-phase linear SISO system having relative degree one can be transformed to the format shown in (1).

The interactions among the subsystems are specified by a directed graph $\mathcal{G}$ which consists of a set of vertices denoted by $\mathcal{V}=\{1, \cdots, N\}$ and a set of edges denoted by $\mathcal{E}$. A vertex denotes a subsystem, and the edge $(i, j)$ in the edge set $\mathcal{E}$ means that subsystem $i$ is a neighbor of subsystem $j$ and subsystem $j$ can obtain the output information $y_{i}$ from subsystem $i$ but not necessarily vice versa. A path from vertex $i_{1}$ to vertex $i_{l}$ is a sequence of ordered edges of the form $\left(i_{k}, i_{k+1}\right), k=1, \cdots, l-1$. A directed graph is strongly connected if there is a directed path from every vertex to every other vertex. For the graph $\mathcal{G}$, its adjacency matrix $A$ is defined such that $a_{i i}=0, a_{i j}=1$ if $(j, i) \in \mathcal{E}$ and $a_{i j}=0$ otherwise. The Laplacian matrix $L$ is defined as $l_{i i}=\sum_{j=1}^{N} a_{i j}$ and $l_{i j}=-a_{i j}$ when $i \neq j$.

Throughout this section, we suppose the interactions among the subsystems satisfy the following assumption.

Assumption 2 The directed graph $\mathcal{G}$ is strongly connected, i.e., the corresponding Laplacian matrix $L$ is irreducible (i.e., there does not exist a permutation matrix $T$ such that $T L T^{T}$ is block triangular [15]). 
Under Assumption 2, the Laplacian matrix $L$ has the following properties.

Lemma 1 ([17]) Zero is a simple eigenvalue of $L$ with $\mathbf{1}$ (a column vector with all entries equal to one) as a right eigenvector and all nonzero eigenvalues have positive real parts.

Lemma 2 ([13]) Suppose that $\mathcal{G}$ is strongly connected. Let $r=\left[r_{1}, \cdots, r_{N}\right]^{T}$ be the positive left eigenvector of $L$ associated with the zero eigenvalue and $R=$ $\operatorname{diag}\left(r_{1}, \cdots, r_{N}\right)$. Then, $\widehat{L} \triangleq R L+L^{T} R$ is the symmetric Laplacian matrix associated with an undirected connected graph. Let $\xi$ be any vector with positive entries. Then, $\min _{\xi^{T} x=0, x \neq 0} \frac{x^{T} \widehat{L} x}{x^{T} x}>\frac{\lambda_{2}(\widehat{L})}{N}$, where $\lambda_{2}(\widehat{L})$ denotes the smallest nonzero eigenvalue of $\widehat{L}$.

The objective of this section is to solve the adaptive consensus control problem, i.e., to design an adaptive consensus protocol using the relative output information $y_{i}-y_{j}, i \neq j$, of neighboring subsystems to ensure that $\lim _{t \rightarrow \infty}\left\|y_{i}(t)-y_{j}(t)\right\|=0$ and $\lim _{t \rightarrow \infty}\left\|z_{i}(t)-z_{j}(t)\right\|=$ $0, \forall i, j=1, \cdots, N$.

\subsection{Control Design}

We define the following variables:

$$
\begin{aligned}
\varrho_{i} & =\sum_{j=1}^{N} a_{i j}\left(y_{i}-y_{j}\right), \\
\nu_{i} & =\sum_{j=1}^{N} a_{i j}\left(z_{i}-z_{j}\right), \quad i=1, \cdots, N .
\end{aligned}
$$

Let $\varrho=\left[\varrho_{1}, \cdots, \varrho_{N}\right]^{T}, \nu=\left[\nu_{1}^{T}, \cdots, \nu_{N}^{T}\right]^{T}, y=$ $\left[y_{1}, \cdots, y_{N}\right]^{T}$, and $z=\left[z_{1}^{T}, \cdots, z_{N}^{T}\right]^{T}$. Then, $(2)$ can be rewritten in a compact form as

$$
\begin{aligned}
& \varrho=L y \\
& \nu=\left(L \otimes I_{n-1}\right) z,
\end{aligned}
$$

where $\otimes$ denotes the Kronecker product of matrices $L$ and $I_{n-1}$, defined as

$$
L \otimes I_{n-1}=\left[\begin{array}{ccc}
l_{11} I_{n-1} & \cdots & l_{1 N} I_{n-1} \\
\vdots & \ddots & \vdots \\
l_{N 1} I_{n-1} & \cdots & l_{N N} I_{n-1}
\end{array}\right] .
$$

Since the graph $\mathcal{G}$ is strongly connected, it is well known via Lemma 1 that the consensus problem is solved if and only if $\varrho$ and $\nu$ asymptotically converge to zero.
Based on the relative output information of neighboring subsystems, we design a distributed consensus protocol to each subsystem as

$$
u_{i}=-\left(k_{i}+\varphi_{i}\right) \varrho_{i}
$$

where $\varphi_{i}=\varrho_{i}^{2}$ and $k_{i}$ is the adaptive gain, generated by

$$
\dot{k}_{i}=\gamma \varrho_{i}^{2}, \text { with } k_{i}(0)=k_{0}
$$

where $\gamma>0$ and $k_{0}$ is any known positive constant.

Using (4) for (1), we can obtain the closed-loop dynamics of the network as

$$
\begin{aligned}
& \dot{\varrho}=\left(I_{N} \otimes h^{T}\right) \nu+\left(I_{N} \otimes a\right) \varrho-b L(K+\varphi) \varrho, \\
& \dot{\nu}=\left(I_{N} \otimes B\right) \nu+\left(I_{N} \otimes d\right) \varrho,
\end{aligned}
$$

where $K=\operatorname{diag}\left(k_{1}, \cdots, k_{N}\right)$ and $\varphi=\operatorname{diag}\left(\varphi_{1}, \cdots, \varphi_{N}\right)$.

The following is the main result of this section.

Theorem 3 Suppose that Assumptions 1 and 2 hold. The distributed protocol (4) together with the adaptive law (5) solve the consensus control problem for the unknown linear subsystems described by (1). Moreover, the adaptive gains $k_{i}$ will converge to some finite steady-state values.

\section{Proof Let}

$$
V_{\varrho}=\frac{1}{2} \sum_{i=1}^{N} r_{i}\left(2 k_{i}+\varphi_{i}\right) \varrho_{i}^{2}+\frac{\lambda_{2}(\widehat{L}) b}{8 N \gamma} \sum_{i=1}^{N}\left(k_{i}-k^{*}\right)^{2}
$$

where $r \triangleq\left[r_{1}, \cdots, r_{N}\right]^{T}$ is the positive left eigenvector of $L$ associated with the zero eigenvalue, $k^{*}$ is a constant to be determined later, $\lambda_{2}(\widehat{L})$ denotes the smallest nonzero eigenvalue of $\widehat{L} \triangleq R L+L^{T} R$, where $R \triangleq \operatorname{diag}\left(r_{1}, \cdots, r_{N}\right)>0$.

Using (6) and (5), we can obtain the time derivative of $V_{\varrho}$ as

$$
\begin{aligned}
\dot{V}_{\varrho}= & \sum_{i=1}^{N} 2 r_{i}\left(k_{i}+\varphi_{i}\right) \varrho_{i} \dot{\varrho}_{i}+\sum_{i=1}^{N} r_{i} \varrho_{i}^{2} \dot{k}_{i} \\
& +\frac{\lambda_{2}(\widehat{L}) b}{4 N \gamma} \sum_{i=1}^{N}\left(k_{i}-k^{*}\right) \dot{k}_{i} \\
= & -b \varrho^{T}(K+\varphi) \widehat{L}(K+\varphi) \varrho+2 \varrho^{T} R(K+\varphi) \\
& \times\left[\left(I_{N} \otimes h^{T}\right) \nu+\left(I_{N} \otimes a\right) \varrho\right]+\gamma \varrho^{T} \varphi R \varrho \\
& +\frac{\lambda_{2}(\widehat{L}) b}{4 N}\left(\varrho^{T} K \varrho-k^{*} \varrho^{T} \varrho\right) .
\end{aligned}
$$


Let $\bar{\varrho}=(K+\varphi) \varrho$. By the definitions of $\varrho$ and $\varrho$, we have

$$
\varrho^{T}\left[\left(K^{-1}+\varphi^{-1}\right) r\right]=\varrho^{T} r=y^{T} L^{T} r=0,
$$

where we have used fact that $r^{T} L=0$. Since every entry of $r$ is positive, it is easy to see that every entry of $\left(K^{-1}+\right.$ $\left.\varphi^{-1}\right) r$ is also positive. In light of Lemma 2 , we get that

$$
\begin{aligned}
\varrho^{T}(K+\varphi) \widehat{L}(K+\varphi) \varrho & >\frac{\lambda_{2}(\widehat{L})}{N} \bar{\varrho}^{T} \varrho \\
& =\frac{\lambda_{2}(\widehat{L})}{N}\|(K+\varphi) \varrho\|^{2} .
\end{aligned}
$$

Substituting (9) into (8) and using the Young's inequality [2], we have

$$
\begin{aligned}
\dot{V}_{\varrho}< & -s\|(K+\varphi) \varrho\|^{2}+\frac{s}{4}\|(K+\varphi) \varrho\|^{2}+\frac{s}{8}\|K \varrho\|^{2} \\
& +\frac{s}{8}\|\varphi \varrho\|^{2}-\frac{s}{4} k^{*}\|\varrho\|^{2}+\frac{8}{s}\left\|R\left(I_{N} \otimes h^{T}\right)\right\|^{2}\|\nu\|^{2} \\
& +\left(\frac{8}{s}\left\|R\left(I_{N} \otimes a\right)\right\|^{2}+\frac{2 \gamma^{2}}{s}\|R\|^{2}+\frac{s}{8}\right)\|\varrho\|^{2} \\
\leq & -\frac{s}{4} k^{*}\|\varrho\|^{2}+\frac{8}{s}\left\|R\left(I_{N} \otimes h^{T}\right)\right\|^{2}\|\nu\|^{2} \\
& +\left(\frac{8}{s}\left\|R\left(I_{N} \otimes a\right)\right\|^{2}+\frac{2 \gamma^{2}}{s}\|R\|^{2}+\frac{s}{8}\right)\|\varrho\|^{2},
\end{aligned}
$$

where $s=\frac{\lambda_{2}(\widehat{L}) b}{N}$.

Since $B$ is Hurwitz, there exists a positive definite matrix $P$ such that $P B+B^{T} P=-(m+2) I$, where $m=$ $\frac{8}{s}\left\|R\left(I_{N} \otimes h^{T}\right)\right\|^{2}$. Let

$$
V_{\nu}=\nu^{T}\left(I_{N} \otimes P\right) \nu .
$$

The time derivative of $V_{\nu}$ along (6) can be obtained as

$$
\begin{aligned}
\dot{V}_{\nu} & =-(m+2)\|\nu\|^{2}+2 \nu^{T}\left(I_{N} \otimes P d\right) \varrho \\
& =\nu^{T}\left[I_{N} \otimes\left(P B+B^{T} P\right)\right] \nu+2 \nu^{T}\left(I_{N} \otimes P d\right) \varrho \\
& \leq-(m+1)\|\nu\|^{2}+\left\|I_{N} \otimes P d\right\|^{2}\|\varrho\|^{2} .
\end{aligned}
$$

Finally, consider the following Lyapunov function candidate

$$
V_{1}=V_{\varrho}+V_{\nu} \text {. }
$$

Note that in $(7), r_{i}>0, \varphi_{i}(t) \geq 0, k_{i}(t) \geq k_{0}>0$. Then, it is easy to see that $V_{1}$ is positive definite with respect to $\varrho, \nu$, and $k_{i}-k^{*}, i=1, \cdots, N$. Choose

$k^{*}=\frac{4}{s}\left[\alpha+\frac{8}{s}\left\|R\left(I_{N} \otimes a\right)\right\|^{2}+\frac{2 \gamma^{2}}{s}\|R\|^{2}+\frac{s}{8}+\left\|I_{N} \otimes P d\right\|^{2}\right]$,

where $\alpha$ is a positive constant. Therefore, from (10) and (11), we can obtain that

$$
\dot{V}_{1}=-\alpha\|\varrho\|^{2}-\|\nu\|^{2}
$$

Since $\dot{V}_{1} \leq 0, V_{1}(t)$ is bounded and so are $\varrho, \nu$, and each $k_{i}$. By noting that $k_{i}$ are monotonically increasing, it then follows that the adaptive gains $k_{i}$ converge to some finite values. Note that $\dot{V}_{1} \equiv 0$ implies that $\varrho=0$ and $\nu=0$. Applying the LaSalle's Invariance principle [6], we can conclude that $\lim _{t \rightarrow \infty} \varrho(t)=0$ and $\lim _{t \rightarrow \infty} \nu(t)=0$, which implies that the adaptive consensus problem is solved.

Remark 2 The adaptive consensus protocol (4) depends only on the relative output information of neighboring subsystems and thereby are fully distributed. A distinct feature of the distributed protocol (4) is that it include a term $\varphi_{i}$ to provide additional design flexibility, which is vital to ensure that (4) is applicable to any strongly connected directed graph. For undirected and connected graphs, it can be shown that the adapitve protocol without $\varphi_{i}$, i.e., $u_{i}=-k_{i} \varrho_{i}$, solves the consensus problem. The derivation can be completed by following similar steps in the proof of Theorem 3, which is omitted for brevity.

Remark 3 It should be noted that a distributed adaptive protocol is also presented for linear subsystems with directed graphs in [11]. Compared to the previous work [11], the result in this section has at least two contributions. First, the subsystem dynamics considered in this section can be unknown, contrary to the subsystems in [11] which are precisely known linear systems. Second, in contrast to the adaptive protocol in [11] which relies on the relative states of neighboring subsystems, the adaptive protocol proposed in this section depends on only the relative outputs of neighboring subsystems, which clearly is more challenging to design.

\section{Distributed Output Tracking with an Arbi- trary Constant Reference}

In the previous section, a distributed adaptive protocol is designed to achieve consensus for the subsystems in (1) with strongly connected directed graphs. The final consensus value reached by the subsystems, which depends on the initial values and the subsystem dynamics, might be unknown a priori. In some cases, it might be desirable for the subsystem states or outputs to converge onto a reference signal. This is usually called distributed tracking or leader-follower consensus. In this section, we consider the problem that the outputs of the group of $N$ subsystems in (1) are required to track a constant reference signal.

\subsection{Problem Statement}

We define the output tracking errors as

$$
e_{i}=y_{i}-y_{0}, \quad i=1, \cdots, N,
$$


where $y_{0} \in \mathbb{R}$ is an arbitrary constant reference signal. In our setup, not every subsystem has access to $y_{0}$, and the output tracking will be achieved through the interactions among the subsystems. We use a diagonal matrix $\Delta=$ $\operatorname{diag}\left(\delta_{11}, \cdots, \delta_{N N}\right)$ to denote the access to $y_{0}$ in the way that if $\delta_{i i}=1$, the $i$-th subsystem has access to the value of $y_{0}$ for the control design, and $\delta_{i i}=0$ otherwise. At least one subsystem has the access to $y_{0}$.

The distributed output tracking problem considered in this section is to design a distributed controller using the relative output information $y_{i}-y_{j}, i \neq j$, of neighboring subsystems to ensure the convergence to zero of output tracking errors $e_{i}$ for $i=1, \cdots, N$, i.e., the convergence of the subsystem outputs $y_{i}$ to the common value $y_{0}$.

Regarding the interactions among the subsystems and the reference signal $y_{0}$, we make the following assumption.

Assumption 3 The directed graph $\mathcal{G}$ among the $N$ subsystems is strongly connected and at least one subsystem has access to $y_{0}$.

For notational convenience, we let $Q=L+\Delta$. Under Assumption 3, it is not difficult to verify that $Q$ is a nonsingular $M$-matrix [15], which satisfies the following property.

Lemma $4([\mathbf{1 1}, \mathbf{1 5}])$ There exists a positive diagonal matrix $G$ such that $G Q+Q^{T} G \geq r_{0} I$ for some positive constant $r_{0}$. Specifically, such $a G$ is given by $\operatorname{diag}\left(g_{1}, \ldots, g_{N}\right)$, where $\left[g_{1}, \cdots, g_{N}\right]^{T}=\left(Q^{T}\right)^{-1} \mathbf{1}$.

\subsection{Control Design}

We define

$$
\zeta_{i}=\sum_{j=1}^{N} a_{i j}\left(y_{i}-y_{j}\right)+\delta_{i i}\left(y_{i}-y_{0}\right), \quad i=1, \cdots, N
$$

Using the notation $q_{i j}$ to denote the $(i, j)$-th entry of the matrix $Q$, we have

$$
\begin{aligned}
\zeta_{i} & =\sum_{j=1}^{N} a_{i j}\left(e_{i}-e_{j}\right)+\delta_{i i} e_{i} \\
& =\sum_{j=1}^{N} q_{i j} e_{j} .
\end{aligned}
$$

Let

$$
\begin{aligned}
\bar{z} & =-B^{-1} d y_{0}, \\
\eta & =-\frac{1}{b}\left(h^{T} \bar{z}+a y_{0}\right) .
\end{aligned}
$$

Then, the subsystem dynamics in (1) can be rewritten as

$$
\begin{aligned}
& \dot{e}_{i}=h^{T} \tilde{z}_{i}+a e_{i}+b\left(u_{i}-\eta\right), \\
& \dot{\tilde{z}}_{i}=B \tilde{z}_{i}+d e_{i},
\end{aligned}
$$

where $\tilde{z}_{i}=z_{i}-\bar{z}$.

We design the distributed controller to each subsystem:

$$
u_{i}=-\left(f_{i}+\rho_{i}\right) \zeta_{i}+\xi_{i},
$$

where $\rho_{i}=\zeta_{i}^{2}, f_{i}$ and $\xi_{i}$ are generated by

$$
\begin{gathered}
\dot{f}_{i}=\gamma \zeta_{i}^{2}, \text { with } f_{i}(0)=f_{0}, \\
\dot{\xi}_{i}=-\xi_{i}+u_{i},
\end{gathered}
$$

with $f_{0}$ being any known positive constant.

Note that $f_{i}$ in (16) can be viewed as an adaptive gain, and the dynamics of $\xi_{i}$ in (17) is considered as an internal model for tracking a constant.

Theorem 5 Suppose Assumptions 1 and 3 hold. For the $N$ subsystems in (1), the distributed controller specified by (15) together with the adaptive law (16) and the internal model (17) solve the distributed output tracking problem.

Proof Let

$$
\tilde{\eta}_{i}=\eta-\xi_{i}+\frac{1}{b} e_{i}
$$

The dynamics of $\tilde{\eta}_{i}$ can be obtained as

$$
\dot{\tilde{\eta}}_{i}=-\tilde{\eta}_{i}+\frac{a+1}{b} e_{i}+\frac{1}{b} h^{T} \tilde{z}_{i}
$$

It follows from (14) that the closed-loop subsystem dynamics can be written as

$$
\dot{e}_{i}=h^{T} \tilde{z}_{i}+a e_{i}-b\left(f_{i}+\rho_{i}\right) \zeta_{i}+b\left(\xi_{i}-\eta\right) .
$$

With $\xi_{i}-\eta=\frac{1}{b} e_{i}-\tilde{\eta}_{i}$, we have

$$
\dot{e}_{i}=-b\left(f_{i}+\rho_{i}\right) \zeta_{i}+h^{T} \tilde{z}_{i}+(a+1) e_{i}-b \tilde{\eta}_{i} .
$$

Let

$$
V_{\zeta}=\sum_{i=1}^{N} \frac{1}{2} g_{i}\left(2 f_{i}+\rho_{i}\right) \zeta_{i}^{2}+\frac{1}{2 \gamma} \sum_{i=1}^{N}\left(f_{i}-f^{*}\right)^{2}
$$

where $g_{i}$ is defined as in Lemma 4 and $f^{*}$ is a constant 
to be determined later. Using (19) and (16), we have

$$
\begin{aligned}
\dot{V}_{\zeta}= & \sum_{i=1}^{N} 2 g_{i}\left(f_{i}+\rho_{i}\right) \zeta_{i} \sum_{j=1}^{N} q_{i j} \dot{e}_{j}+\sum_{i=1}^{N} g_{i} \rho_{i} \dot{f}_{i} \\
& +\frac{1}{\gamma} \sum_{i=1}^{N}\left(f_{i}-f^{*}\right) \dot{f}_{i} \\
= & -b \zeta^{T}(F+\rho)\left(G Q+Q^{T} G\right)(F+\rho) \zeta \\
& +2 \zeta^{T} G(F+\rho)\left[M_{1} \tilde{z}+M_{2} \tilde{\eta}+(a+1) Q e\right] \\
& +\gamma \zeta^{T} \rho G \zeta+\zeta^{T} F \zeta-f^{*} \zeta^{T} \zeta,
\end{aligned}
$$

where $\zeta=\left[\zeta_{1}^{T}, \cdots, \zeta_{N}^{T}\right]^{T}, \tilde{z}=\left[\tilde{z}_{1}^{T}, \cdots, \tilde{z}_{N}^{T}\right]^{T}, \tilde{\eta}=$ $\left[\tilde{\eta}_{1}^{T}, \cdots, \tilde{\eta}_{N}^{T}\right]^{T}, e=\left[e_{1}^{T}, \cdots, e_{N}^{T}\right]^{T}, F=\operatorname{diag}\left(f_{1}, \cdots, f_{N}\right)$, $\rho=\operatorname{diag}\left(\rho_{1}, \cdots, \rho_{N}\right), M_{1}=Q \otimes h^{T}$, and $M_{2}=-b Q$.

Note that $\zeta=Q e$. From (20) and the Young's inequality, we have

$$
\begin{aligned}
\dot{V}_{\zeta} \leq & -\frac{1}{2} r\|(F+\rho) \zeta\|^{2}-f^{*}\|\zeta\|^{2} \\
& +\frac{10}{r}\left[\frac{\gamma^{2}}{4}\|G\|^{2}\|\zeta\|^{2}+\frac{1}{4}\|\zeta\|^{2}+\left\|M_{1} G\right\|^{2}\|\tilde{z}\|^{2}\right. \\
& \left.+\left\|M_{2} G\right\|^{2}\|\tilde{\eta}\|^{2}+(a+1)^{2}\|\zeta\|^{2}\right] \\
= & -\frac{1}{2} r\|(F+\rho) \zeta\|^{2}-f^{*}\|\zeta\|^{2}+\frac{10}{r}\left[\left(\frac{\gamma^{2}}{4}\|G\|^{2}+\frac{1}{4}\right.\right. \\
& \left.\left.+(a+1)^{2}\right)\|\zeta\|^{2}+\left\|M_{1} G\right\|^{2}\|\tilde{z}\|^{2}+\left\|M_{2} G\right\|^{2}\|\tilde{\eta}\|^{2}\right],
\end{aligned}
$$

where $r=b r_{0}$.

Since $B$ is Hurwitz, there exists a positive definite matrix $P$ such that $P B+B^{T} P=-3 I$. Let

$$
V_{z}=\sum_{i=1}^{N} \tilde{z}_{i}^{T} P \tilde{z}_{i}
$$

From (14), it can be obtained that

$$
\begin{aligned}
\dot{V}_{z} & =-3 \sum_{i=1}^{N}\left\|\tilde{z}_{i}\right\|^{2}+2 \sum_{i=1}^{N} \tilde{z}_{i}^{T} P d e_{i} \\
& \leq-2\|\tilde{z}\|^{2}+\|P d\|^{2}\|e\|^{2} \\
& \leq-2\|\tilde{z}\|^{2}+\|P d\|^{2}\left\|Q^{-1}\right\|^{2}\|\zeta\|^{2} .
\end{aligned}
$$

Let

$$
V_{\eta}=\sum_{i=1}^{N} \tilde{\eta}_{i}^{2} .
$$

From (18), it can be obtained that

$$
\begin{aligned}
\dot{V}_{\eta} & =-2 \sum_{i=1}^{N} \tilde{\eta}_{i}^{2}+2 \sum_{i=1}^{N} \tilde{\eta}_{i}\left(\frac{a+1}{b} e_{i}+\frac{1}{b} h^{T} \tilde{z}_{i}\right) \\
& \leq-\sum_{i=1}^{N} \tilde{\eta}_{i}^{2}+2 \sum_{i=1}^{N}\left[\frac{(a+1)^{2}}{b^{2}} e_{i}^{2}+\frac{\|h\|^{2}}{b^{2}}\left\|\tilde{z}_{i}\right\|^{2}\right] \\
& \leq-\|\tilde{\eta}\|^{2}+2 \frac{(a+1)^{2}}{b^{2}}\left\|Q^{-1}\right\|^{2}\|\zeta\|^{2}+2 \frac{\|h\|^{2}}{b^{2}}\|\tilde{z}\|^{2} .
\end{aligned}
$$

Finally, let

$$
V_{2}=V_{\zeta}+2 \beta_{1} V_{\eta}+\beta_{2} V_{z}
$$

where we set

$$
\begin{aligned}
& \beta_{1}=\frac{10}{r}\left\|M_{2} G\right\|^{2} \\
& \beta_{2}=4 \beta_{1} \frac{\|h\|^{2}}{b^{2}}+\frac{10}{r}\left\|M_{1} G\right\|^{2},
\end{aligned}
$$

and

$$
\begin{aligned}
f^{*}= & \beta_{3}+\frac{10}{r}\left[\frac{\gamma^{2}}{4}\|G\|^{2}+\frac{1}{4}+(a+1)^{2}\right] \\
& +4 \beta_{1} \frac{(a+1)^{2}}{b^{2}}\left\|Q^{-1}\right\|^{2}+\beta_{2}\|P d\|^{2}\left\|Q^{-1}\right\|^{2},
\end{aligned}
$$

where $\beta_{3}$ is a positive constant. Then, from (21), (22) and (23), we can obtain that

$$
\dot{V}_{2} \leq-\beta_{3}\|\zeta\|^{2}-\beta_{2}\|\tilde{z}\|^{2}-\beta_{1}\|\tilde{\eta}\|^{2} \leq 0 .
$$

Therefore we can conclude that the variables $\zeta, \tilde{z}, \tilde{\eta}$, and $F$ are bounded. Similar to the proof of Theorem 3, we can conclude that $\lim _{t \rightarrow \infty} \zeta(t)=0, \lim _{t \rightarrow \infty} \tilde{z}(t)=0$, and $\lim _{t \rightarrow \infty} \tilde{\eta}(t)=0$, which implies that $\lim _{t \rightarrow \infty} e_{i}(t)=0$ for $i=1, \cdots, N$, i.e., the distributed output tracking problem is solved.

Remark 4 Similar to the adaptive protocol (4), the adaptive controller (15) is also distributed, depending on only the relative output information. Different from (4), the adaptive controller (15) includes an internal model, which is vital to tracking the constant reference signal.

Remark 5 Note that the distributed controller (15) together with the adaptive law (16) and the internal model (17) are able to ensure that the outputs of the $N$ subsystems in (1) asymptotically track the output of the leader described by

$$
\begin{aligned}
& \dot{y}_{0}=h^{T} z_{0}+a y_{0}+b u_{0}, \\
& \dot{z}_{0}=B z_{0}+d y_{0}
\end{aligned}
$$


with a constant control input $u_{0}$. The derivations are similar to those in the proof of Theorem 5.

Remark 6 In the previous related work [25], controllers are designed to ensure that a group of heterogeneous agents with nonlinear dynamics reach an agreement which is governed by specified reference dynamics. Differen$\mathrm{t}$ from [25] where the design of the controllers requires global information of the Laplacian matrix, the effort of this paper is to design fully distributed adaptive protocols for a network of unknown linear subsystems.

Remark 7 For the distributed tracking problem in this section, it is shown in Theorem 5 that $\zeta$ and $\tilde{z}$ are bounded, which, by using (13), (14) and noting the boundedness of $y_{0}$, implies that the subsystems' state variables $y_{i}$ and $z_{i}$ are also bounded. For the leaderless consensus in the previous section, the final consensus value, which is a solution to each subsystem with $u_{i}=0$ and thereby depends on the subsystem dynamics, is not necessarily bounded, so are $y_{i}$ and $z_{i}$. We need to impose conditions on the subsystems' state matrix $\left[\begin{array}{cc}h^{T} & a \\ B & d\end{array}\right]$ to guarantee the boundedness of $y_{i}$ and $z_{i}$ for the leaderless case. For instance, if the subsystems' state matrix have simple eigenvalues on the imaginary axis and does not have eigenvalues with positive real parts, $y_{i}$ and $z_{i}$ are bounded.

\section{Simulation Example}

In this section, a simulation example is provided to validate the effectiveness of the theoretical results.

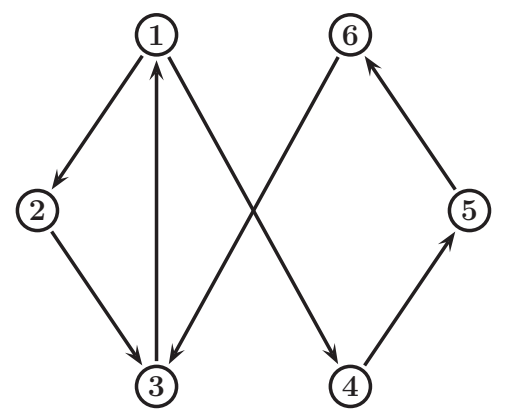

Fig. 1. A directed communication graph.

Consider a network of six third-order subsystems, described by (1), with

$$
B=\left[\begin{array}{ll}
-1 & 1 \\
-2 & 0
\end{array}\right], d=\left[\begin{array}{l}
0 \\
1
\end{array}\right], h=\left[\begin{array}{l}
0 \\
1
\end{array}\right], a=-0.5, b=3
$$

These parameters are given for running the simulation. They will not be used for controller design. The interaction graph among the six subsystems is given as in Fig. 1, which satisfies Assumption 2. To illustrate Theorem
3 , the distributed adaptive consensus protocol is chosen as

$$
\begin{aligned}
& u_{i}=-\left(k_{i}+\zeta_{i}^{2}\right) \zeta_{i}, \\
& \dot{k}_{i}=\gamma \zeta_{i}^{2},
\end{aligned}
$$

where $\gamma=5$ and the initial states of $f_{i}$ are randomly chosen within the interval $[1,3]$. The subsystem states $y_{i}$ and $z_{i}$ are depicted in Figs. 2 and 3, from which it can be observed that consensus is indeed achieved. The adaptive gains $k_{i}, i=1, \cdots, 6$ are shown in Fig. 4, which converge to some steady-state values.

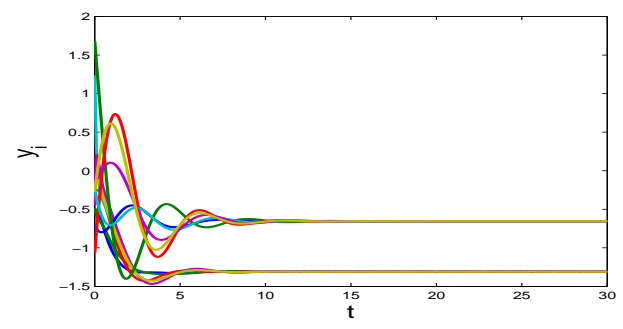

Fig. 2. The subsystem states $y_{i}, i=1, \cdots, 6$.

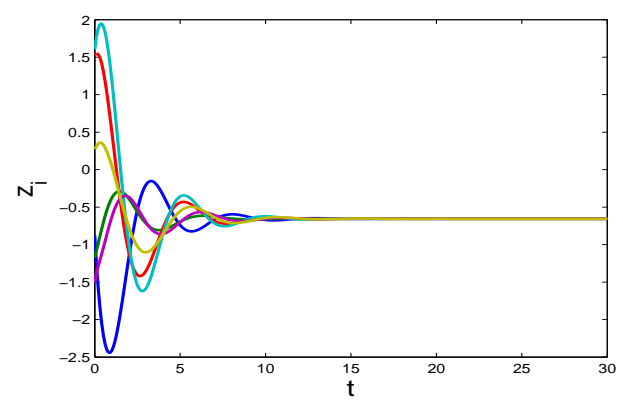

Fig. 3. The subsystem states $z_{i}, i=1, \cdots, 6$.

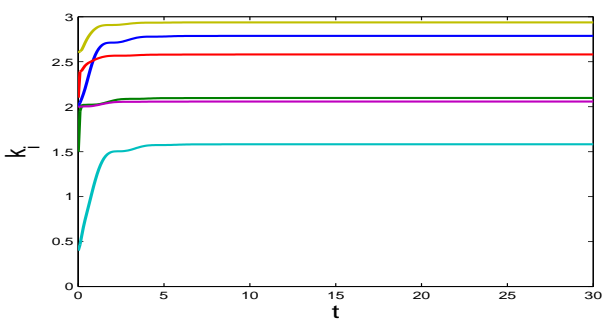

Fig. 4 . The adaptive gains $k_{i}, i=1, \cdots, 6$.

To illustrate Theorem 5 , let the reference signal $y_{0}=5$. Assume that only the subsystem 1 has access to $y_{0}$ and thereby $\delta_{11}=1, \delta_{i i}=0, i=2, \cdots, 6$. According Theorem 5 , the distributed adaptive controller is designed as

$$
\begin{aligned}
u_{i} & =-\left(f_{i}+\zeta_{i}^{2}\right) \zeta_{i}+\xi_{i}, \\
\dot{f}_{i} & =\gamma \zeta_{i}^{2} \\
\dot{\xi}_{i} & =-\left(f_{i}+\zeta_{i}^{2}\right) \zeta_{i}
\end{aligned}
$$

with $\gamma=5$. The simulation results in this case are shown in Figs. 5 and 6 . Fig. 5 shows that the output tracking 
errors $e_{i}=y_{i}-y_{0}, i=1, \cdots, 6$, converge to zero and Fig. 6 demonstrates that the adaptive gains $f_{i}, i=1, \cdots, 6$, converge to finite constant values.

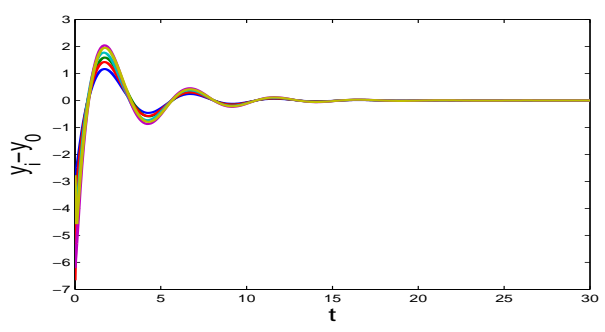

Fig. 5. The output tracking errors $y_{i}-y_{0}, i=1, \cdots, 6$.

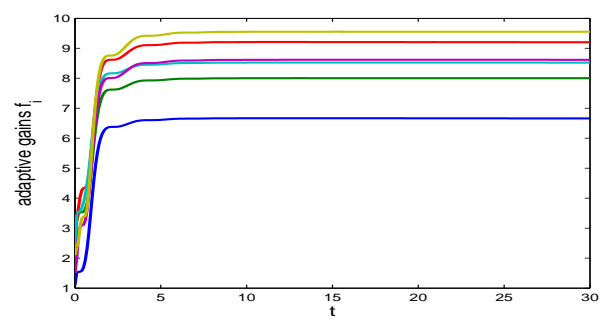

Fig. 6 . The adaptive gains $f_{i}, i=1, \cdots, 6$.

\section{Conclusion}

In this paper, we have considered both the distributed leaderless consensus and the output tracking problems of a group of unknown linear subsystems with directed graphs. Two distributed adaptive protocols have been presented, respectively, to achieve leaderless consensus and output tracking in the presence of an arbitrary constant reference signal. The proposed adaptive protocols rely on only the relative outputs of neighboring subsystems and hence are fully distributed. Comparing with the existing results on distributed adaptive protocols, the results in this paper extends the distributed adaptive schemes to the case with unknown linear parameter and the relative output information.

\section{References}

[1] G. Antonelli. Interconnected dynamic systems: An overview on distributed control. IEEE Control Systems Magazine, 33(1):76-88, 2013.

[2] D.S. Bernstein. Matrix Mathematics: Theory, Facts, and Formulas. Princeton University Press, 2009.

[3] M. Guo and D.V. Dimarogonas. Consensus with quantized relative state measurements. Automatica, 49(8):2531 - 2537, 2013.

[4] Y.G. Hong, G.R. Chen, and L. Bushnell. Distributed observers design for leader-following control of multi-agent networks. Automatica, 44(3):846-850, 2008.

[5] H.K. Khalil. Nonlinear Systems. Prentice Hall, Englewood Cliffs, NJ, 2002.
[6] M. Krstić, I. Kanellakopoulos, and P. Kokotovic. Nonlinear and Adaptive Control Design. John Wiley \& Sons, New York, 1995.

[7] Z.K. Li, Z.S. Duan, and G.R. Chen. Dynamic consensus of linear multi-agent systems. IET Control Theory and Applications, 5(1):19-28, 2011.

[8] Z.K. Li, Z.S. Duan, G.R. Chen, and L. Huang. Consensus of multiagent systems and synchronization of complex networks: A unified viewpoint. IEEE Transactions on Circuits and Systems I: Regular Papers, 57(1):213-224, 2010.

[9] Z.K. Li, W. Ren, X.D. Liu, and M.Y. Fu. Consensus of multi-agent systems with general linear and Lipschitz nonlinear dynamics using distributed adaptive protocols. IEEE Transactions on Automatic Control, 58(7):1786-1791, 2013.

[10] Z.K. Li, W. Ren, X.D. Liu, and L.H. Xie. Distributed consensus of linear multi-agent systems with adaptive dynamic protocols. Automatica, 49(7):1986-1995, 2013.

[11] Z.K. Li, G.H. Wen, Z.S. Duan, and W. Ren. Designing fully distributed consensus protocols for linear multi-agent systems with directed graphs. IEEE Transactions on Automtic Control, in press, 20104.

[12] C.Q. Ma and J.F. Zhang. Necessary and sufficient conditions for consensusability of linear multi-sgent systems. IEEE Transactions on Automatic Control, 55(5):1263-1268, 2010.

[13] J. Mei, W. Ren, and J. Chen. Consensus of second-order heterogeneous multi-agent systems under a directed graph. In The 2014 American Control Conference, pages 802-807, 2014.

[14] R. Olfati-Saber and R.M. Murray. Consensus problems in networks of agents with switching topology and time-delays. IEEE Transactions on Automatic Control, 49(9):1520-1533, 2004.

[15] Z. Qu, Cooperative Control of Dynamical Systems: Applications to Autonomous Vehicles. London, UK: SpringerVerlag, 2009.

[16] W. Ren. On consensus algorithms for double-integrator dynamics. IEEE Transactions on Automatic Control, 53(6):1503-1509, 2008.

[17] W. Ren and R.W. Beard. Consensus seeking in multiagent systems under dynamically changing interaction topologies. IEEE Transactions on Automatic Control, 50(5):655-661, 2005.

[18] W. Ren, R.W. Beard, and E.M. Atkins. Information consensus in multivehicle cooperative control. IEEE Control Systems Magazine, 27(2):71-82, 2007.

[19] J.H. Seo, H. Shim, and J. Back. Consensus of high-order linear systems using dynamic output feedback compensator: Low gain approach. Automatica, 45(11):2659-2664, 2009.

[20] H.S. Su, G.R. Chen, X.F. Wang, and Z.L. Lin. Adaptive second-order consensus of networked mobile agents with nonlinear dynamics. Automatica, 47(2):368-375, 2011.

[21] S.E. Tuna. Conditions for synchronizability in arrays of coupled linear systems. IEEE Transactions on Automatic Control, 54(10):2416-2420, 2009.

[22] W.W. Yu, W. Ren, W.X. Zheng, G.R. Chen, and J.H. Lü. Distributed control gains design for consensus in multi-agent systems with second-order nonlinear dynamics. Automatica, 49(7):2107-2115, 2013.

[23] H.W. Zhang, F.L. Lewis, and A. Das. Optimal design for synchronization of cooperative systems: State feedback, observer, and output feedback. IEEE Transactions on Automatic Control, 56(8):1948-1952, 2011. 
[24] H.W. Zhang, F.L. Lewis, and Z.H. Qu. Lyapunov, adaptive, and optimal design techniques for cooperative systems on directed communication graphs. IEEE Transactions on Industrial Electronics, 59(7):3026-3041, 2012.

[25] L.J. Zhu and Z.Y. Chen. Robust homogenization and consensus of nonlinear multi-agent systems. Systems and Control Letters, 65:50-55, 2014. 